\title{
Performance Analysis of gradient decent adaptive filters for noise cancellation in Signal Processing
}

\author{
Darshana Kundu (Phd Scholar), Dr. Geeta Nijhawan (Prof.) ECE Dept, \\ Manav Rachna International University, Faridabad, Haryana, India
}

\begin{abstract}
Adaptive filtering is a very active area of research in the field of signal processing. Adaptive noise cancellation using adaptive filtering is an alternative technique of estimating signals corrupted by additive noise or interference. As received signal is corrupted by noise continuously where both received signal and noise are continuous signal, then a need of adaptive filter arises. This paper presents a comparative study of the various gradient decent based adaptive algorithms-recursive least square (RLS),least mean square (LMS) along with various categories of LMS such as normalized LMS(NLMS) Signum LMS(SLMS), fast block LMS(FBLMS) based noise canceller. The performance comparison of simulated model of noise canceller is presented in figures which shows the error signal, amplitudes of actualization coefficients and transmission characteristics.
\end{abstract}

Keywords: Adaptive noise cancellation (ANC), LMS, RLS, SLMS, FBLMS, NLMS algorithms, FIR

\section{INTRODUCTION}

Adaptive filtering techniques are used in a wide range of applications such as noise cancelation, echo cancelation. Noise cancelation is a common occurrence in today telecommunication systems. Adaptive filters are used especially for non-stationary signals and environments. Application of adaptive filters includes adaptive noise cancellation, which is used to filter out noise or interference signals from noisy speech signal in real life environment. Adaptive noise Cancellation is an alternative technique of estimating signals corrupted by additive noise or interference. It needs two inputs - a primary input containing the corrupted signal and a reference input containing noise signal correlated in some unknown way with the primary noise. The reference input is adaptively filtered and subtracted from the primary input to obtain the signal estimate.

\subsection{SYSTEM DESIGN}

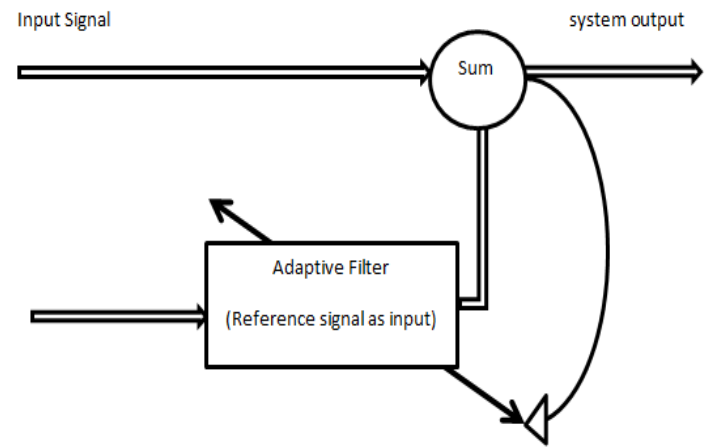

Fig: 1 Basic block diagram of adaptive filter used as noise canceller
As shown above, an adaptive filter consists of two parts: a linear filter and an adaptive algorithm. You can use linear filters with different filter types, such as finite impulse response (FIR) and infinite impulse response (IIR) filters. Where $u(n)$ is the input signal to a linear filter at time $\mathrm{n}, \mathrm{y}(\mathrm{n})$ is the corresponding output signal, $d(n)$ is another input signal to the adaptive filter ,e(n) is the error signal that denotes the difference between $\mathrm{d}(\mathrm{n})$ and $\mathrm{y}(\mathrm{n}), W_{i}(\mathrm{n})$ is the multiplicative gain and also is known as the filter coefficient whereas ' $l$ ' is an integer with a value range of $[0, N-1]$ where $N$ is the length of the filter. The adaptive algorithm adjusts $W_{i}(\mathrm{n})$ iteratively to minimize the error signal e(n).

$\xi(\mathrm{n})=\mathrm{E}[\mathrm{e}(\mathrm{n})]$.......(1)

The error estimation $\mathrm{e}(\mathrm{n})$ is

$\mathrm{e}(\mathrm{n})=\mathrm{d}(\mathrm{n})-\mathrm{y}(\mathrm{n})$

$y(n)=w(n) * u(n) \quad \ldots \ldots .(3)$

where $u(n)$ is an input signal vector

The rest of the paper is organized as follows:

In section 2 various adaptive algorithms which are used to train adaptive filters to minimize noise for a deigned system are described. In section 3, simulation results are discussed in detail. Section 4 gives the conclusion \& future aspects.

\section{ADAPTIVE ALGORITHMS}

We can apply different algorithms to the adaptive filter to control how the filter adjusts their coefficients. The adaptive algorithms adjust the filter coefficients to minimize the mean square error (MSE) as cost function. 


\subsection{LMS(standard LMS) algorithm:-}

The least-mean square (LMS) algorithm updates the linear filter coefficients such that the mean square error (MSE) cost function is minimized .It perform the following operation to update coefficients of the adaptive filter.

a. Calculates the error signal $e(n)$ by using equation(2).

b. Coefficient updating equation is

$w(n+1)=w(n)+\mu u(n) e(n)$,

Where $\mu$ is the step size of the adaptive filter (n) is weight vector and $u(n)$ is the input signal vector.

\subsection{Signum LMS}

Some adaptive filter based applications require to implement adaptive filter algorithms on hardware targets, such as digital signal processing (DSP) devices. These targets require a simplified version of the standard LMS algorithm. The signum function, as defined by the following equation, can simplify the standard LMS algorithm.

$\operatorname{sgn}(x)=\left\{\begin{array}{cc}1 ; & x>0 \\ 0 ; & x=0 \\ -1 ; & x<0\end{array}\right.$

Sign-sign LMS algorithm-Applies the signum function to both $e(n)$ and $\mathrm{u}(\mathrm{n})$. This algorithm updates the coefficients of an adaptive filter using the following equation:

$$
\mathrm{W}(\mathrm{n}+1)=\mathrm{w}(\mathrm{n})+\mu \cdot \operatorname{sgn}(\mathrm{e}(\mathrm{n})) \cdot \operatorname{sgn}(\mathrm{u}(\mathrm{n})) .
$$

Notice that when either $e(n)$ or $\mathrm{u}(\mathrm{n})$ is zero, this algorithm does not involve multiplication operations. When neither $e(n)$ or $\mathrm{u}(\mathrm{n})$ is zero, this algorithm involves only one multiplication operation. The sign LMS algorithms involve fewer multiplication operations than other algorithms. When the step size $\mu$ equals a power of 2 , the sign LMS algorithms can replace the multiplication operations with shift operations

\subsection{Normalized LMS}

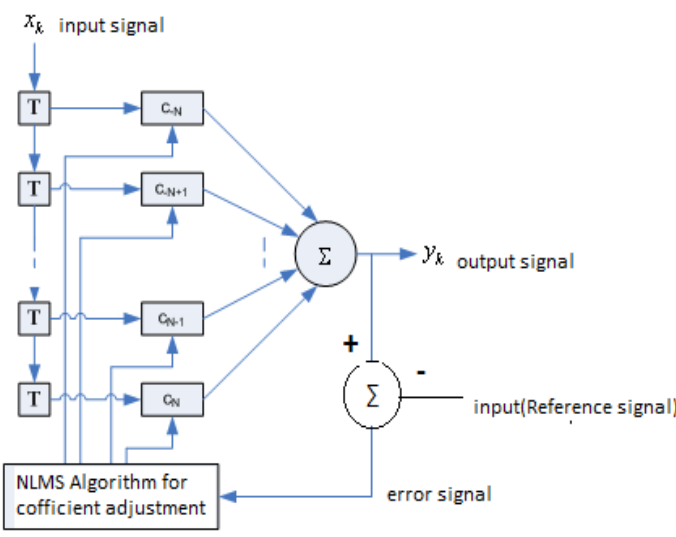

Fig (3):Linear transversal filter with Normalized LMS
The normalized LMS (NLMS) algorithm is a modified form of the standard LMS algorithm. The NLMS algorithm updates the coefficients of an adaptive filter by using the following equation:

$\vec{w}(n+1)=\vec{w}(\mathrm{n})+\mu \mathrm{e}(\mathrm{n}) \cdot \frac{\overleftrightarrow{u(n)}}{\|u(n)\|^{2}} \ldots$

The above equation can be written as:

$\vec{w}(n+1)=\vec{w}(\mathrm{n})+\mu(\mathrm{n}) \cdot \mathrm{e}(\mathrm{n}) \cdot \overrightarrow{u(n)}$

where

$\mu(\mathrm{n})=\frac{\mu}{\|u(n)\|^{2}}$

The NLMS algorithm is similar to standard LMS except that the NLMS algorithm has a time-varying step size $\mu(n)$. This can improve the convergence speed of the adaptive filter.

\subsection{Fast Block LMS}

The adaptive filter applications such as echo cancellation and noise cancellation system require adaptive filters with a large filter length. If the standard LMS algorithm is applied to such adaptive filter, this algorithm might take a long time to complete the filtering and coefficients updating process. This length of time might cause problems in these applications because the adaptive filter must work in real time to filter the input signals. In this situation, the fast block LMS algorithm can be used.

The fast block LMS algorithm uses the fast Fourier transform (FFT) to transform the input signal $x(n)$ to the frequency domain. This algorithm also updates the filter coefficients in the frequency domain. Updating the filter coefficients in the frequency domain can save computational resources. The fast block LMS algorithm differs from the standard LMS algorithm in the following ways:

The fast block LMS algorithm updates the coefficients of an adaptive filter block by block. The block size is exactly the same as the filter length. However, the standard LMS algorithm updates the filter coefficients sample by sample.

The fast block LMS algorithm requires lesser no. of multiplications than the standard LMS algorithm. If both the filter length and block size are $N$, the standard LMS algorithm requires $N(2 N+1)$ multiplications, whereas the fast block LMS algorithm requires only $\left(10 N \log _{2} N+26 N\right)$ multiplications. If $N=1024$, the fast block LMS algorithm can execute 16 times faster than the standard LMS algorithm.

The fast block LMS algorithm calculates the output signal and the error signal before updating the filter coefficients. The following diagram illustrates the steps that this algorithm completes to calculate these signals. 


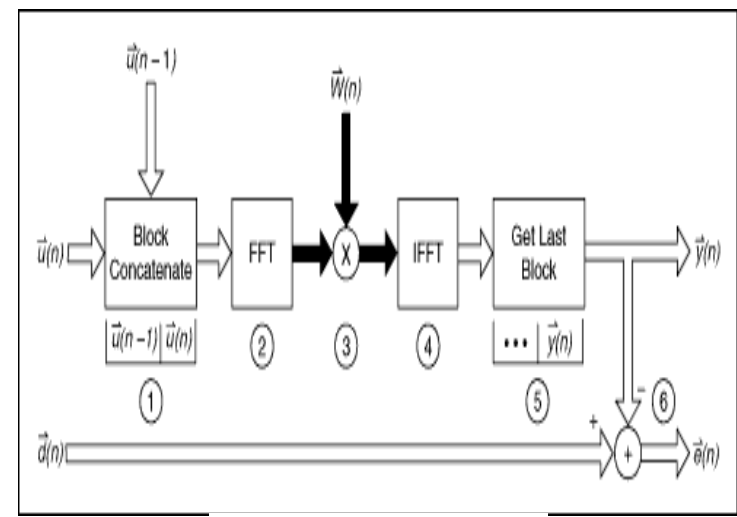

Fig(4): Fast Block LMS

\subsection{RLS Algorithm:}

Recursive least squares (RLS) algorithms exhibit faster convergence speed, and do not have the eigen value spread problem. However, RLS algorithms involve more complicated mathematical operations and require more computational resources than LMS algorithms. RLS algorithm performs the following operations to update the coefficients of an adaptive filter.

1. Calculates the error signal $e(n)$ by using the equation no.(2) as

$e(n)=d(n)-y(n)$.

2. Updates the filter coefficients by using the following equation:

$$
\vec{w}(n+1)=\vec{w}(\mathrm{n})+\mathrm{e}(\mathrm{n}) \cdot \vec{k}(\mathrm{n})
$$

where $\vec{w}(n)$ is the filter coefficients vector and $\vec{K}(n)$ is the gain vector. $\vec{K}(n)$ is defined by the following equation:

$$
\overrightarrow{k(n)}=\frac{p(n) \cdot \overrightarrow{u(n)}}{\lambda+u(n)^{T} \cdot p(n) \cdot \overrightarrow{u(n)}}
$$

where $\lambda$ is the forgetting factor and $P(n)$ is the inverse correlation matrix of the input signal.[adaptive signal theory]

$P(n)$ has the following initial value $P(0)$ :

$$
\mathrm{p}(0)=\left[\begin{array}{ccc}
\delta^{-1} & 0 & 0 \\
0 & \delta^{-1} & 0 \\
0 & 0 & \delta^{-1}
\end{array}\right]
$$

where $\delta$ is the regularization factor. The standard RLS algorithm uses the following equation to update this inverse correlation matrix.

$$
p(n+1)=\lambda^{-1} p(n)-\lambda^{-1} \vec{k}(n) \cdot u(n)^{T} \cdot p(n) \text {. }
$$

\section{SIMULATION RESULTS AND DISCUSSION}

In this section, test results of various noise cancellation systems simulated in MATLAB are presented. The performance comparison is presented in figures, which shows the error signal, amplitudes of actualization coefficients and transmission characteristics of adaptive filtering algorithms based noise cancellers.

\subsection{Different signals of RLS}

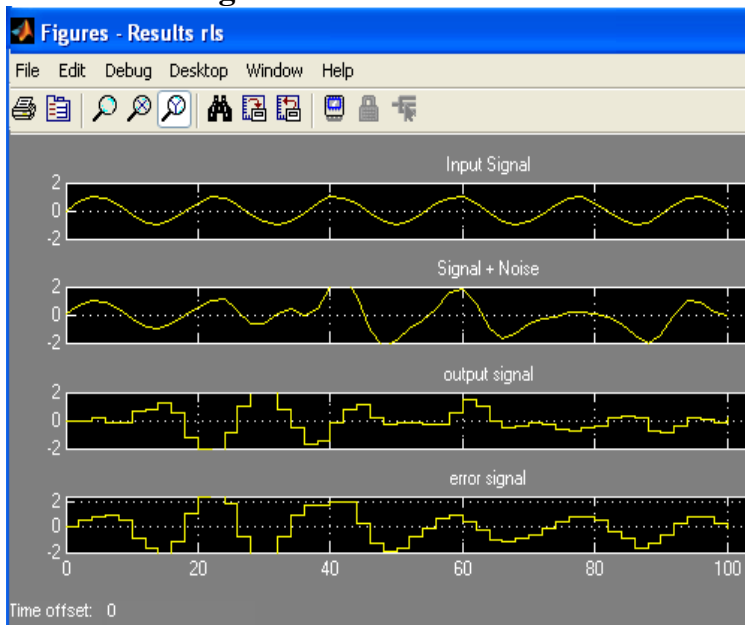

Fig (5): shows input signal, signal+ noise signal, output signal, error signal

\subsection{Different signals of NLMS}

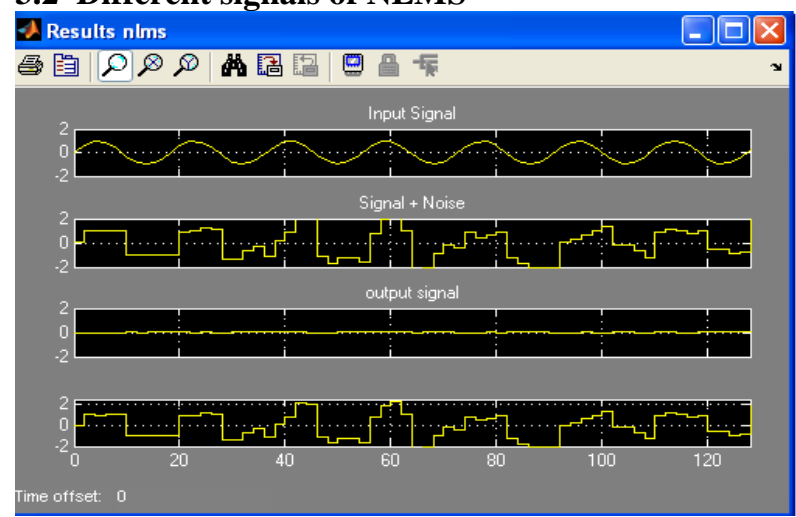

Fig(6): shows input, signal +noise, output \& error signal of normalized LMS

\subsection{Actualization cofficients of various filtering} algorithm 

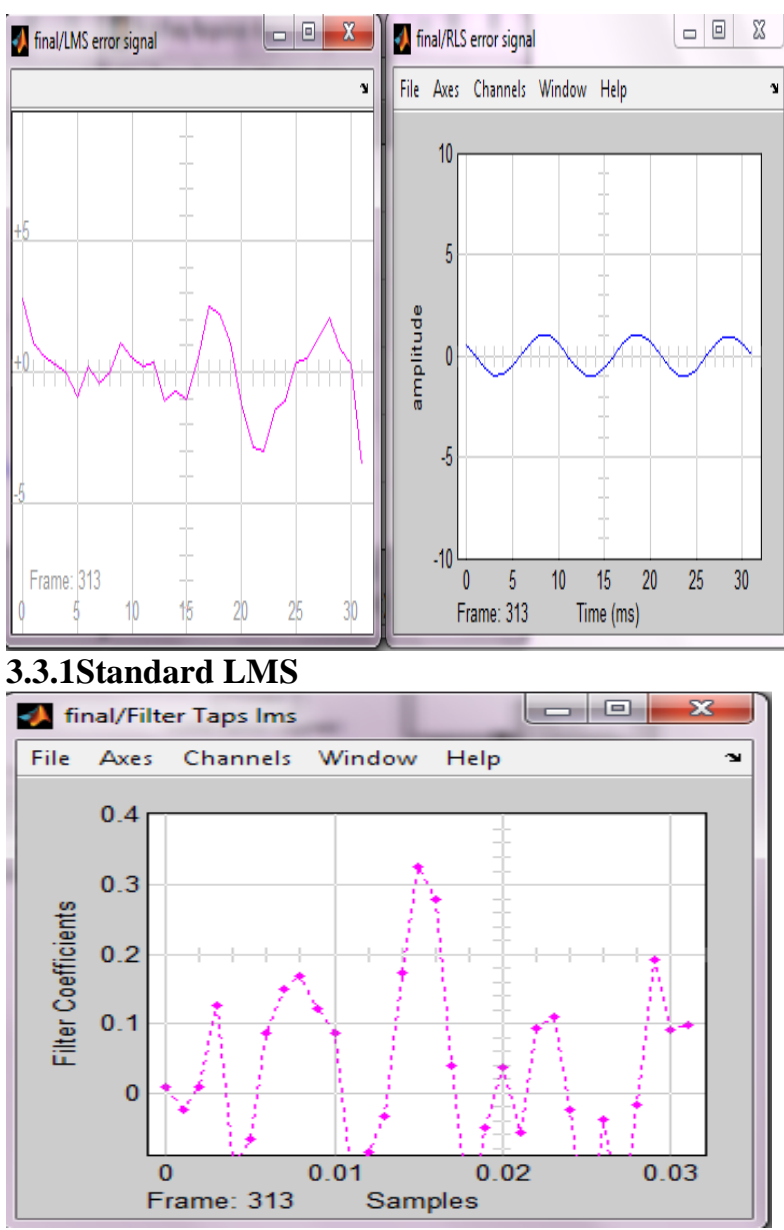

3.3.2 Fast Block LMS

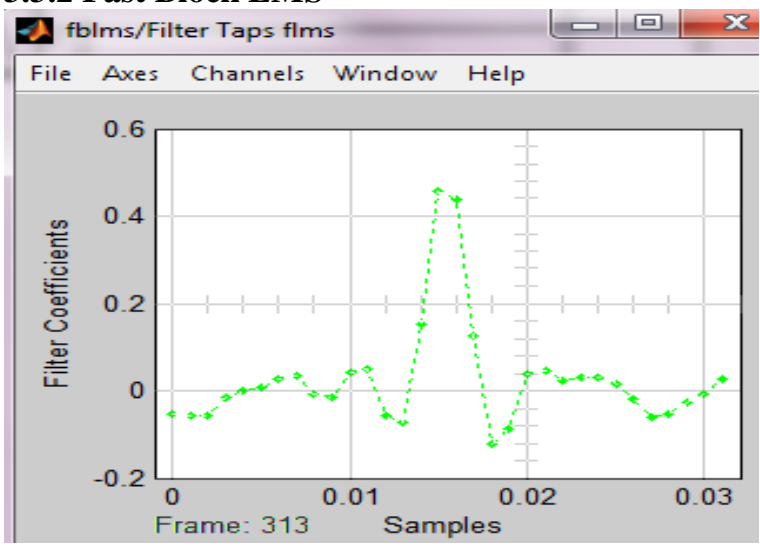

3.3.3 Signum-signum LMS
finaVFilter Taps ssims $\quad-\square \times$

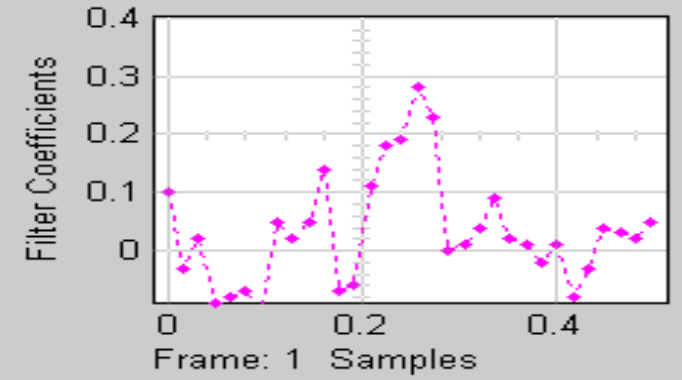

\subsubsection{Normalized LMS \& RLS}

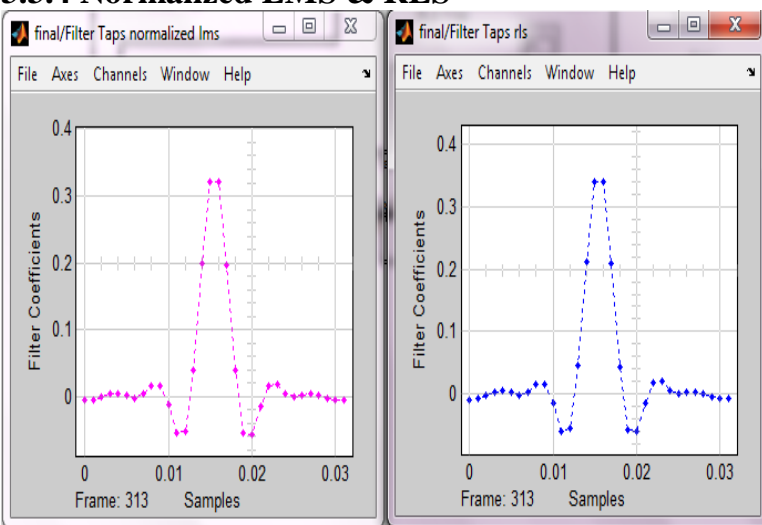

3.4 Error signals of various adaptive filtering algorithms

3.4.1 RLS \& Signum-signum LMS

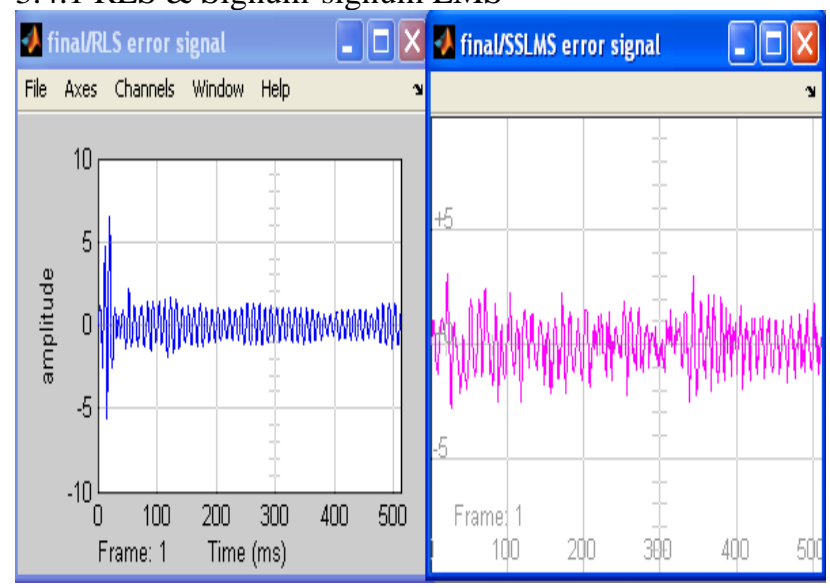

3.4.2 Fast Block LMS 


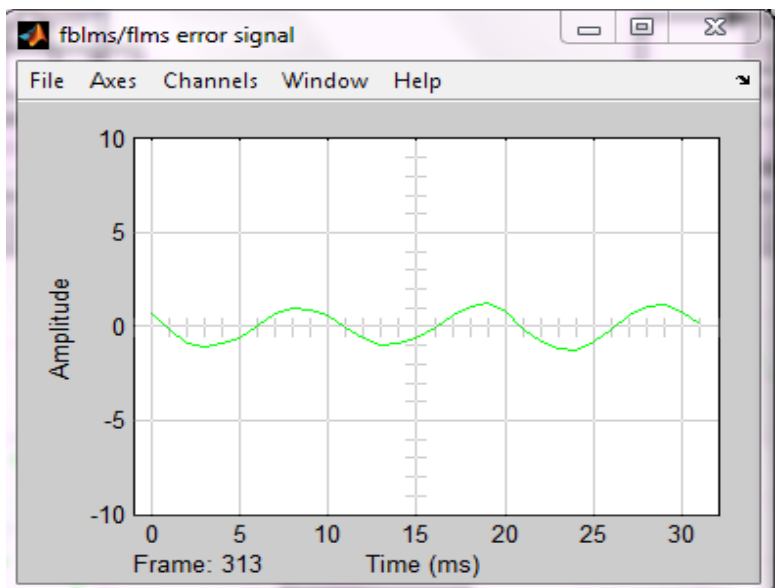

3.4.3 LMS \& RLS

3.5 Transmission characterstics of different adaptive algorithms

3.5.1 Signum-Signum LMS
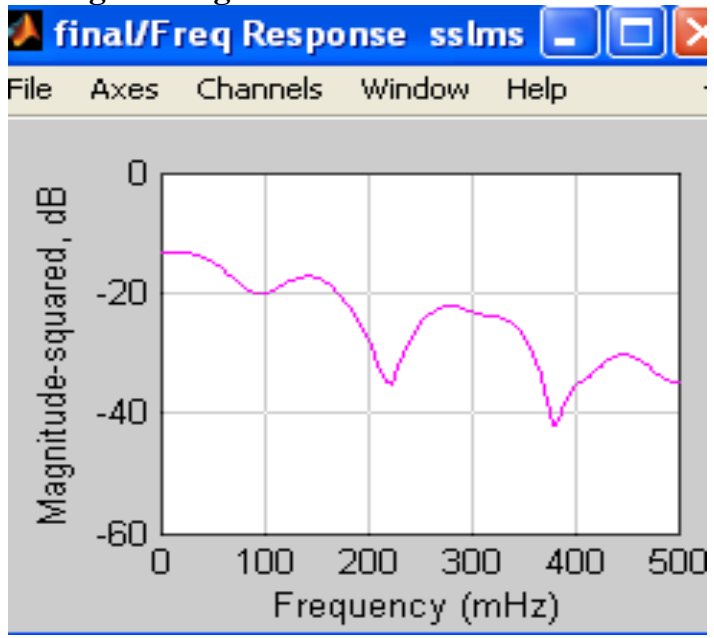

\subsubsection{Fast Block LMS}

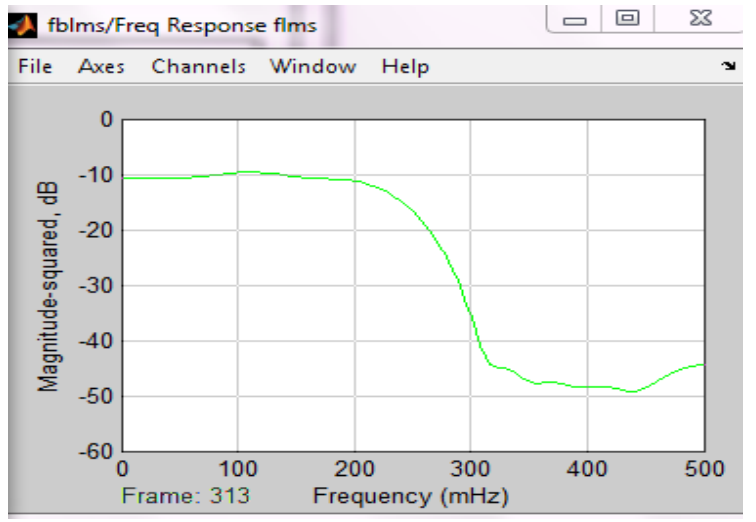

\subsubsection{LMS}

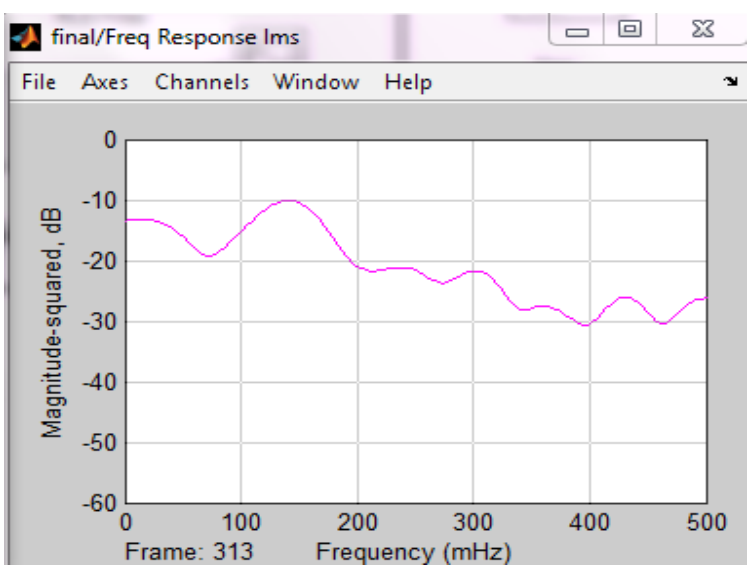

\subsubsection{Signum-signum LMS}
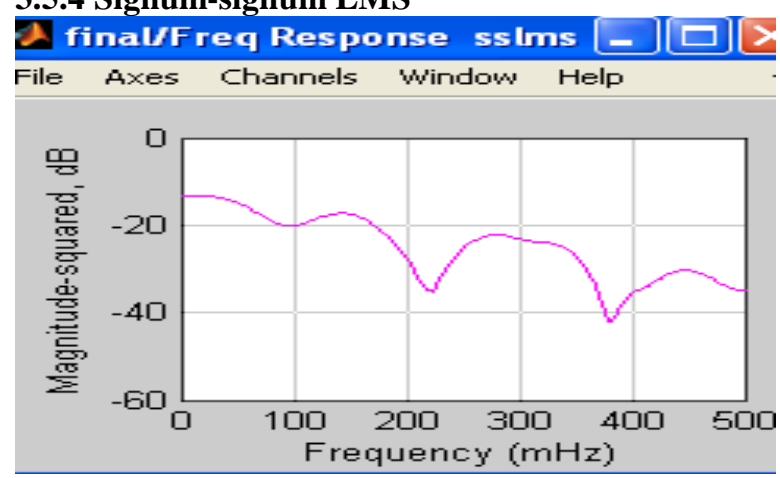

\subsubsection{RLS \& Normalized LMS}

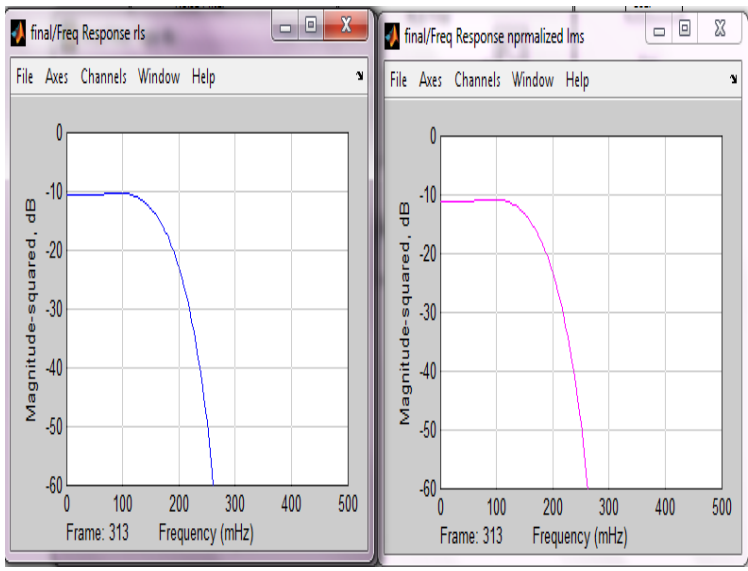

From the simulation results it can be seen that NLMS perform better than LMS whereas the merits of LMS is memory and number of computational operations requirement is less. It has been observed that there is always tradeoff between step size and MSE, the capability of noise cancellation depends on the number of samples taken into consideration. Fast Block LMS is designed to provide similar performance to the standard LMS algorithm while reducing the computational complexity. Simulation shows that Fast Block LMS offer comparable performance with respect to standard LMS and RLS in addition to a large reduction in computation time for higher order filters, Signum Signum LMS algorithm involve very less 
operations as compare to other adaptive algorithms discussed here, as it has only shift and addition operations but it offers slower convergence speed and larger error over standard LMS. RLS based noise canceller also outperform LMS, exhibit faster convergence speed but it requires large computation burden

\section{CONCLUSION AND FUTURE ASPECTS}

In this paper a comparative study of different gradient decent algorithms based adaptive filter known as noise canceller is presented. Here the objective is to optimize different adaptive filtering algorithms so that we can reduce the mean square error to improve received signal fidelity. From the simulation results and discussion it can be concluded that NLMS provided far superior performance over LMS and its variants, but in general it depends on application basically because each one have it's own advantages and disadvantages.RLS also perform better than LMS in terms of noise reduction but computation complexity increases.

The future work includes further optimization of algorithms for all types of noise cancellation and to use the optimized one in the implementation on DSP processors. Further, there are varieties of derivative free optimization techniques such as Genetic algorithm, Particle Swarm Optimization (PSO). In comparison to many other optimization algorithms, the PSO provides advantages of faster convergence rate, strong glol search, simplicity of the algorithm, few adjustal parameters and ease of implementation Therefore can be suitably applied to train the weights adaptive filters.

\section{REFERNCES}

[1]. L.Ferdousel,N.Akhter2,T.Haque "Simulation and Performance Analysis of Adaptive Filtering Algorithms in Noise Cancellation" IJCSI International journal of computer science issues vol.8,issue1,JAN2011 ISSN(online) 16940814

[2]. Vladan Bajic "Design And Implementation Of An Adaptive Noise Canceling System In Wavelet Transform Domain" a thesis presented to the graduate faculty of the university of Akron, august 2005.

[3]. J.Jebastine \& B.Sheela Rani "Comparative Analysis and Design of statistical Examination for various Adaptive Algorithms in speech signal processing”

[4]. Ondrack J., Oravec R., Kadlec J., Cocherova E. "Simulation of RLS and LMS algorithms foradaptive noise cancellation in MATLAB"
[5]. Jashvir Chhikara, Jagbir Singh, "Noise cancellation using adaptive algorithms" IJMER International Journal of Modren Engineering Research, vol.2, Issue3, mayjune2012pp792-795 ISSN:2249-6645.

[6]. Upper Saddle Riven, N.J. "Adaptive Filter Theory".Havkin.S.1996Third Ed., PrenticeHall

[7]. Proakis, "Digital Communication" J.2001 Mc Graw-Hill,NY,4 $4^{\text {th }}$ edition

[8]. Monson H. Hayes "Statistical Digital Signal Processing and Modeling" Wiley-India Edition

[9]. Sharma, O., Janyani, V and Sancheti, S. "Recursive Least Squares Adaptive Filters a better ISI Compensator” 2009 International Journal of Electronics, Circuits and Systems

[10]. B. Widrow, et al., "Adaptive Noise Cancelling: Principles and Applications", Proc. IEEE, vol. 63, pp.1692-1716, Dec. 1975.

[11]. L. Bai and Q. Yin, "A modified nlms algorithm for adaptive noise cancellation," Int. Conf. Acoust., Speech, Signal Processing (ICASSP),pp. 3726 - 3729, 2010. 